\title{
Research Paper \\ Comparing the Prevalence of Constipation Risk Factors in the Elderly With and Without Constipation in Hazrat-e Rasoul (PBUH) Hospital
}

\author{
Rahele Alimoradzadeh ${ }^{1 *}$, Marjan Mokhtare ${ }^{2}$, Shahram Agah²
}

1. Department of Gerontology, School of Medicine, Iran University of Medical Sciences, Tehran, Iran

2. Department of Internal Medicine, School of Medicine, Iran University of Medical Sciences, Tehran, Iran.

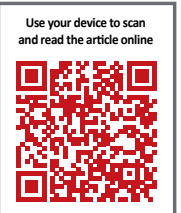

Citeation: Alimoradzadeh R, Mokhtare M, Agah Sh. [Comparing the Prevalence of Constipation Risk Factors in the Elderly With and Without Constipation in Hazrat-e Rasoul (PBUH) Hospital (Persian)]. Iranian Journal of Ageing. 2017; 12(1):78-89. http://dx.doi.org/10.21859/sija-120176

http://dx.doi.org/10.21859/sija-120176

Received: 31 Oct. 2016 Accepted: 21 Feb. 2017

Key words: Constipation, Elderly, Risk factors of constipation

\section{ABSTRACT}

Objectives This study aimed to determine the prevalence and factors associated with constipation among elderly people. Constipation is a common condition affecting elderly people and may lead to complications such as urinary retention and overflow incontinence.

Methods \& Materials This case-control study was conducted on 100 consecutive patients, aged 65 years and older (50 with constipation according to ROME III criteria and 50 without constipation) who were referred to the Gastrointestinal Clinic of Rasoul-e-Akram Hospital. For statistical analysis of the data obtained, SPSS (version 16) for Windows was used.

Results The frequencies of using polypharmacy, calcium channel blocker, anticholinergic drugs, also factors such as immobilization, dehydration, low fiber diet, diabetes, hypothyroidism, stroke, malignancy, renal failure, and Parkinson disease were higher in patients with constipation compared to those without this complaint. There was no significant difference in consumption of opium or having depression.

Conclusion By eliminating the risk factors of constipation, we can reduce constipation and the side effects of using laxatives in the elderly.

\section{Extended Abstract}

\section{Objectives}

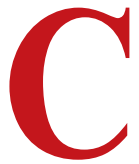

onstipation is defined as difficult or incomplete and irregular bowel movements. According to the literature, constipation is the most common digestive problem in the United States of America, and it is more of a symptom than a disease. This problem can be seen in women and adults older than 65 years old. In the next few years, as Iran will move toward population aging, one of the biggest health problems that could affect this population could be constipation. Notably, knowing the risk factors of constipation is not enough and taking a careful history and reviewing drugs used by the elderly must be considered, too. As the elderly people cannot express their concerns and provide their detailed history while communicating with their doctors due to cognitive and memory problems, it is necessary to conduct a comprehensive assessment irrespective of their chief complaints. In this study, we aimed to determine the risk factors and the prevalence of constipation in the elderly people. Constipation is a common problem among elderly people and may lead to complications such as urinary retention and incontinence overflow if left untreated. Paying more attention to medical history to identify risk factors will pre-

* Corresponding Author:

Rahele Alimoradzadeh, MD

Address: Department of Gerontology, School of Medicine, Iran University of Medical Sciences, Tehran, Iran. Tel: +98 (912) 7299355

E-mail: dr.raal@yahoo.com 
vent the indiscriminate use of laxatives and its side effects in this age group.

\section{Methods \& Materials}

In this study, old people referred to the Clinic of Gastroenterology in Rasoul Akram Hospital in 2015 with complaints of chronic constipation were included. Simultaneously, a group of old people with no complaints of constipation referred for annual examination were recruited as the control group. The relevant information such as age; gender; history of diseases; polypharmacy (more than five drugs); using anticholinergic drugs, opium consumption, non-steroidal antiinflammatory drugs, calcium blockers; inability to move; living in a nursing home; having neurological problems (dementia, Parkinson disease, diabetic neuropathy); depression; dehydration; low fiber diet; metabolic conditions (Hypothyroidism, Hypocalcaemia, Hypokalemia, Uremia, dialysis); mechanical obstruction; lack of privacy and inadequate access to toilet were collected using the checklist. The number of patients studied was 100 . The frequency of the above factors was investigated for the experiment (with constipation) and control groups (without constipation).

Some data were collected by checking medical history and patient's medications, and metabolic conditions were assessed through the application of the relevant tests, physical examination, and if necessary, colonoscopy. To evaluate depression, the depression questionnaire was used. The patients' cognitive and nutritional status was investigated with the help of cognitive tools specific to the elderly people. According to the estimates of diagnostic accuracy equivalent to $96 \%$ of similar studies and considering the maximum effect equal to $3 \%$ in formula, the sample size was determined to be 50 people. The collected data were analyzed with statistical software. In describing the data, the central tendency, frequency, mean, median, and descriptive index, i.e., standard deviation, were used. The study was approved by the Ethics Committee of the University of Medical Sciences (93/D /105/5954). During the study, we were committed to the principles of the Declaration of Helsinki and research ethics legislation.

\section{Results}

In this study, 100 people were evaluated, of them, 50 had constipation problem. Twenty-nine women and 21 men were in the constipation group, and 22 women and 28 men were in the group without constipation. The Chi-square test showed that the gender difference was not significant between two groups. Statistical analysis showed that the difference between the patient group with constipation and diabetes (38\%) and that of the group without constipation but with diabetes $(18 \%)$ was significant. The difference between the two groups in terms of multi-drug consumption was statistically significant $(60 \%$ in the group with constipation, compared with $30 \%$ in patients without constipation). With regard to using calcium channel blocker and anticholinergic drugs, data analysis showed that the difference between two groups was significant. The prevalence of depression and hypothyroidism was also higher in the group of patients with constipation compared to the group of patients without constipation. Regarding the stroke, the Chi-square analysis showed a significant difference between two groups. Eight patients with constipation and one in the control group had a history of malignancy that analysis of data showed significant differences between two groups.

With regard to dialysis, data analysis showed significant differences between two groups (16\% in the group with constipation compared to $2 \%$ in patients without constipation). With regard to drug use, data analysis showed that there was no significant difference between two groups $(24 \%$ in the group with constipation compared with $12 \%$ in patients without constipation). Regarding Parkinson disease, the analysis of data indicated a significant difference between two groups $(12 \%$ in the group with constipation compared to $2 \%$ in patients without constipation). Finally, there was a significant difference between two groups with respect to intake of dietary fiber (64\% in the group with constipation compared to $36 \%$ without constipation).

\section{Conclusion}

The results of this study showed that factors such as taking multiple medications, calcium channel blocker drugs, anticholinergic drugs, lack of movement, dehydration, low fiber diet as well as diseases such as diabetes, hypothyroidism, stroke, malignancies, renal failure, and Parkinson are significantly present in elderly people suffering from constipation compared to those without this problem. On the other hand, the difference in the prevalence of drug use and depression in both groups was not statistically significant. Although constipation is usually considered as a normal part of aging, it is not a disorder that is created by aging itself. Surely changes occur in the digestive system with age, which makes the old people prone to constipation, however, constipation has multi-dimensional etiology. The results of the study by Zahedi et al., which took place in the city of Kerman, showed that the prevalence of constipation increases with age. This has also been confirmed in other studies. The reason for increasing prevalence of constipation in old people may be due to their decreased physical activity, getting food, abdominal muscles atrophy, higher prevalence of mental disorders and taking various drugs. The prevalence of secondary causes increases among the elderly, too. Several factors may be asso- 
ciated with constipation. In this study, we tried to investigate the frequency and risk factors of constipation in old patients. As risk factors of constipation are not well-known due to the lack of specific studies on this age group, this study aimed to emphasize on accurate and comprehensive medical history taking in this age group, irrespective of their chief complaint. Finding the cause of constipation and trying to remove the underlying cause will prevent taking too many laxatives, which leads to the emergence of many complications for elderly people.

\section{Acknowledgments}

This research was extracted from the MD thesis of the first author, in the Department of Gerontology, School of Medicine, Iran University of Medical Sciences, Tehran, Iran.

\section{Conflict of Interest}

The authors declared no conflicts of interest. 


\section{مقايسه فراوانى عوامل خطر بروز يبوست در سالمندان با و بدون يبوست در بيمارستان حضرت

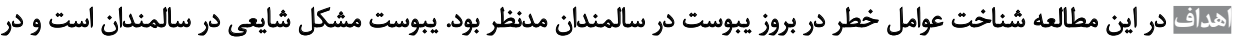

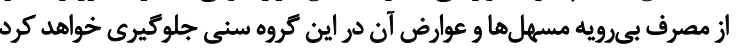

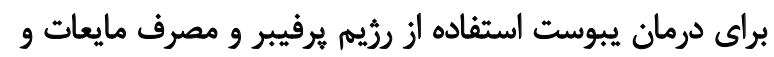

مقدمه

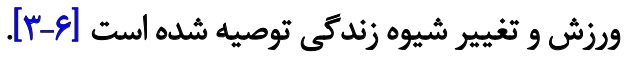

نكته حائز اهميت در تروه سالمندان فقط شناخت عوامل خطر

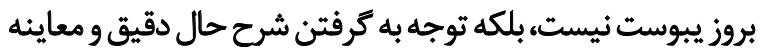

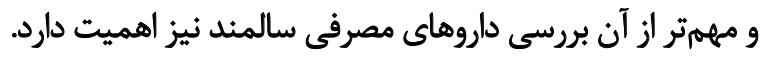

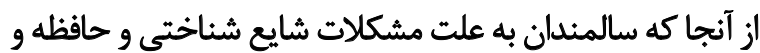

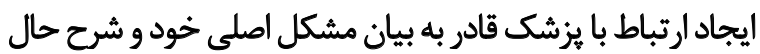

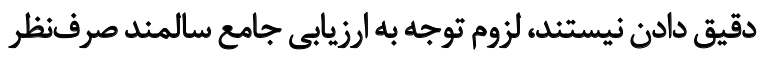

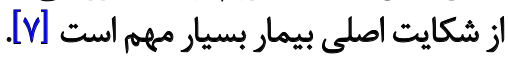

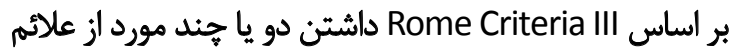

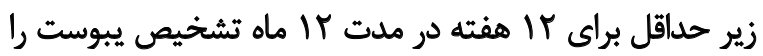

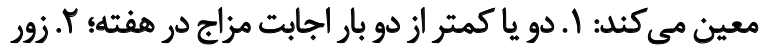

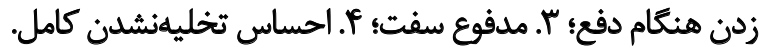

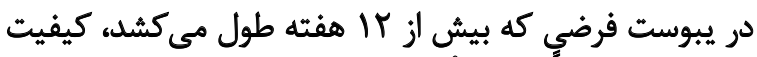

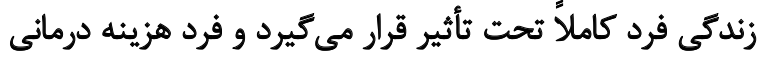

يبوست كه به اجابت مزاج دشوار يا ناكامل و نامنظم اطلاق

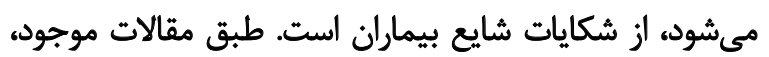

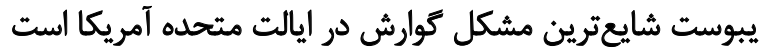

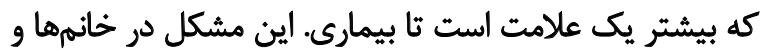

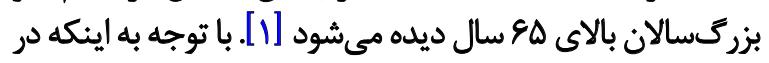

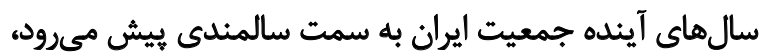

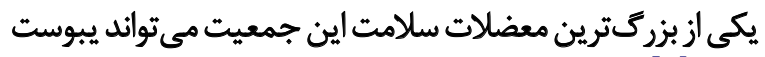

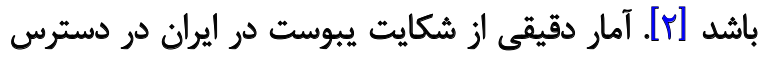

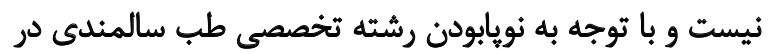

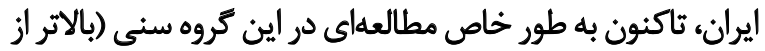

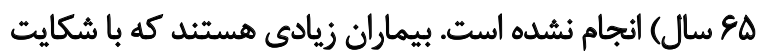

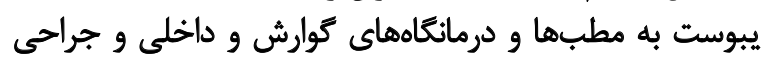

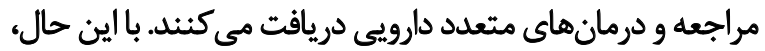
باز هم از اين معضل رنج مىبرند. در تمامى مطالعات انجامشده

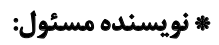

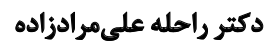

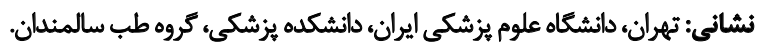

+ تلفن: بست الكثرونيكي: dr.raal@yahoo.com 
خانه سالمندان، مشكلات نورولوزيك (دمانس، ياركينسون،

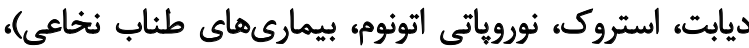

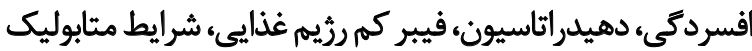

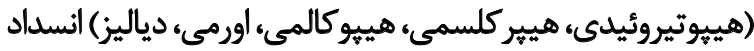

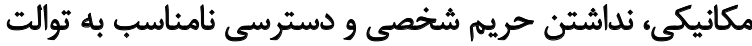
به كمك جكليست جمعآورى شد.

تعداد بيماران بررسىشده صد نفر بود كه در دو ئروه

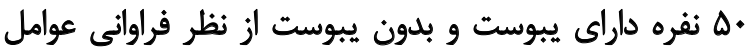

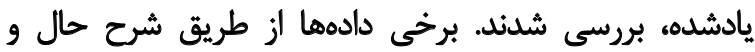

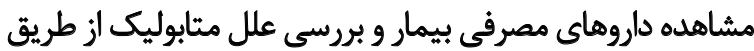

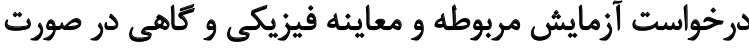

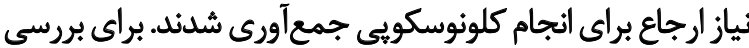

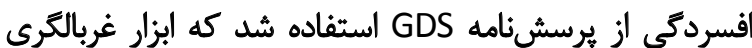

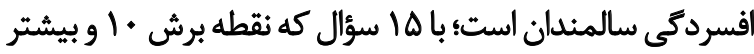

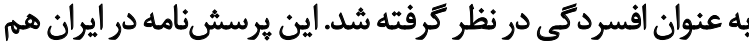

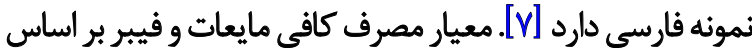

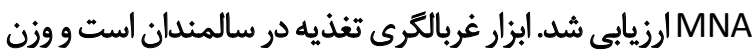

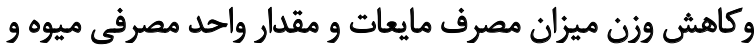

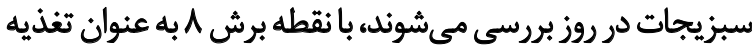

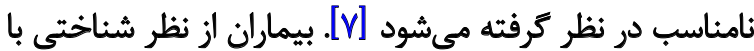

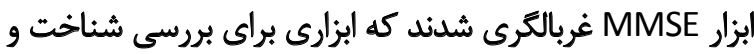

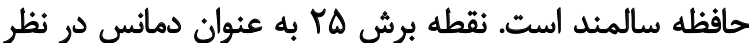

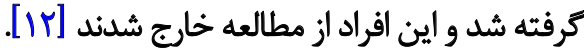
با توجه به برآورد دقت تشخيصى معادل \&9 درصد از مطالعات

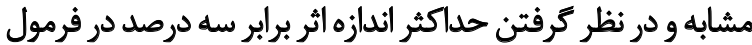

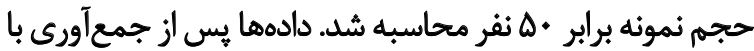

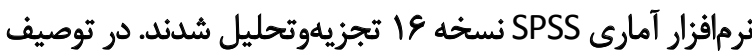

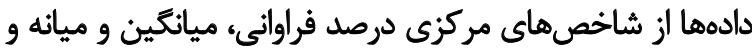

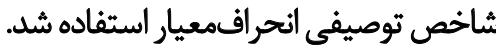

مالاحظاث اخلاقي

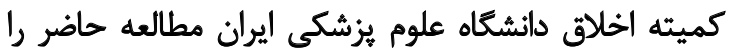

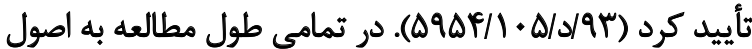

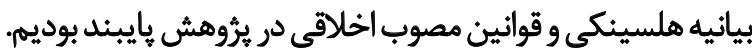

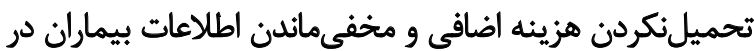

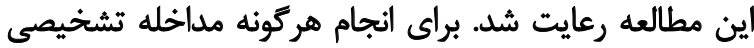

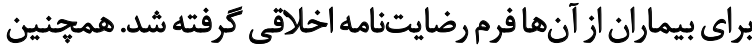

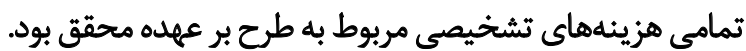

هحدوديتهاى اجهرايي طرح و روث كاهش آنها شركتنكردن بيماران در اقدامات تشخيصى لازم و كاهى ندادن

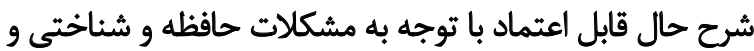
ارتباطى شايع در اين كروه سنى از محدوديتهائ ائه اين مطالعه بودات

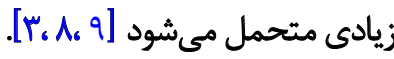

با توجه به درمانهاى مختلف، بيماران هزينه مالى و زمانى زيادى

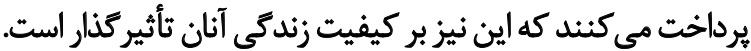

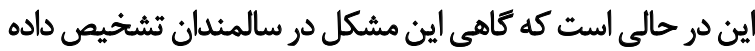

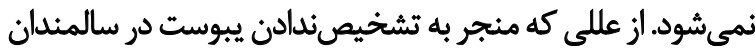

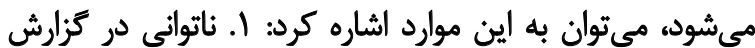

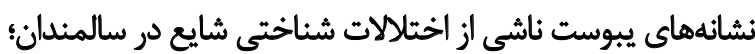

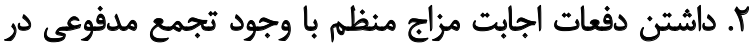

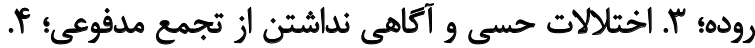

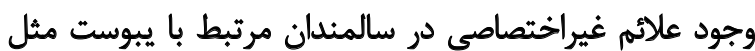
دليريوم، بي اشتهايى و كاهش عملكرد.

يوست با مزمنشدن عوارضى بر جاى مى كذارد كه بـىاختيارى

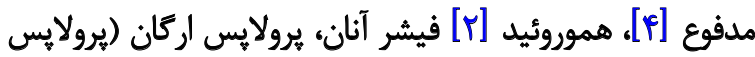

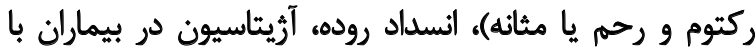

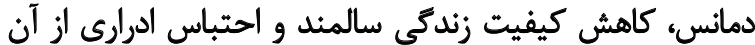

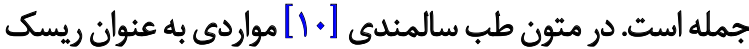

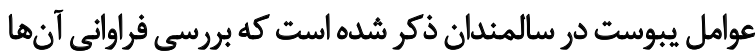

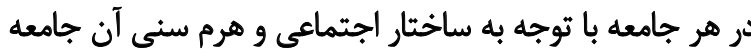

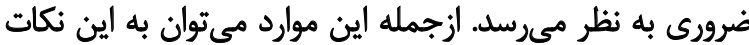

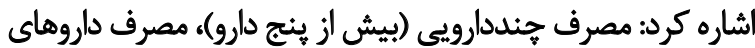

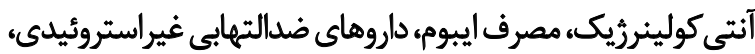

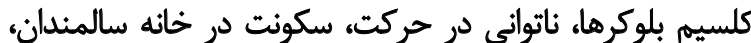

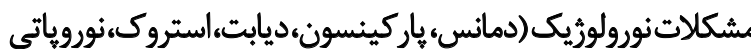

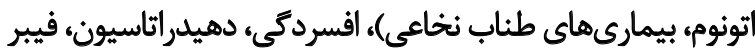

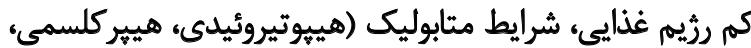

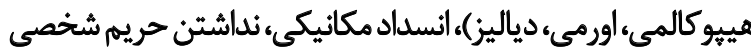

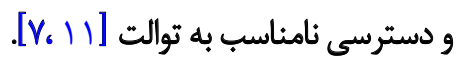

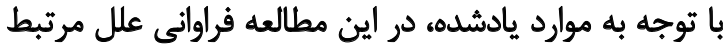

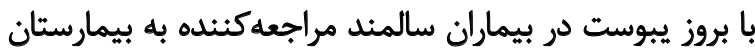

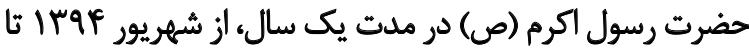

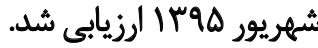
روش مطالعه در اين مطالعه سالمندان مراجعلكنينده به كلينيك كوارش بوري

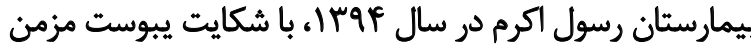

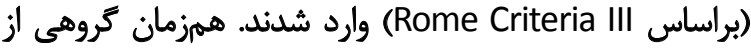
سالمندان بدون شكايت از يبوست نيز كه برائ براي معاينه ساليانه

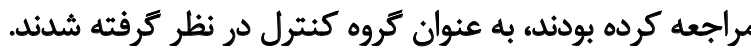

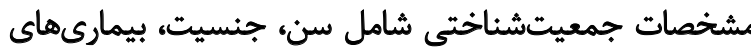

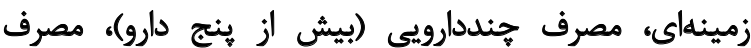

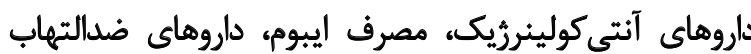

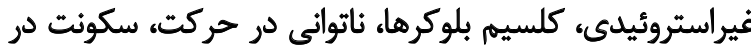




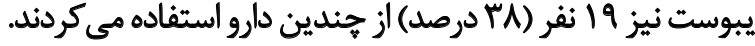

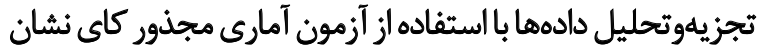

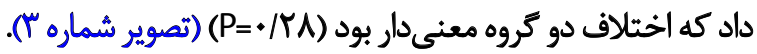
از نظر مصرف داروهاى بلاكر كانال كلسيد در كروه دهر دهار

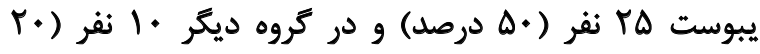

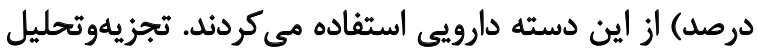

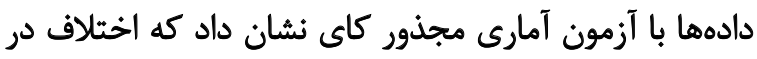

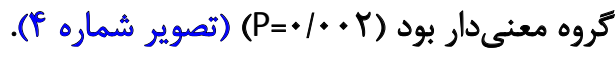
بيست نفر (•f درصد) از افراد دجار يبوست و نيز A نفر

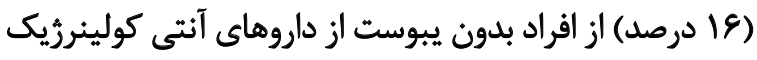

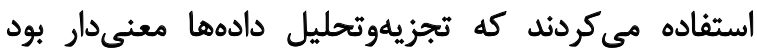

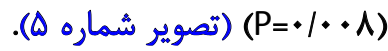

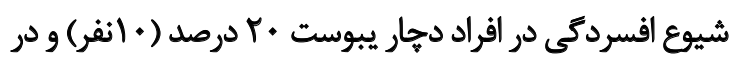

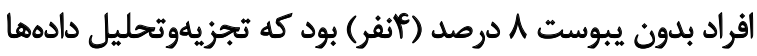

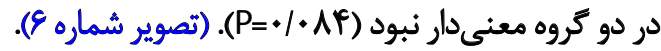

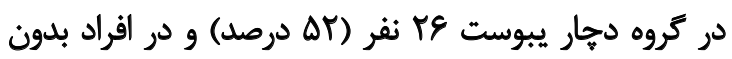

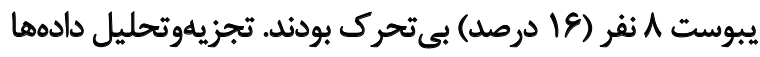
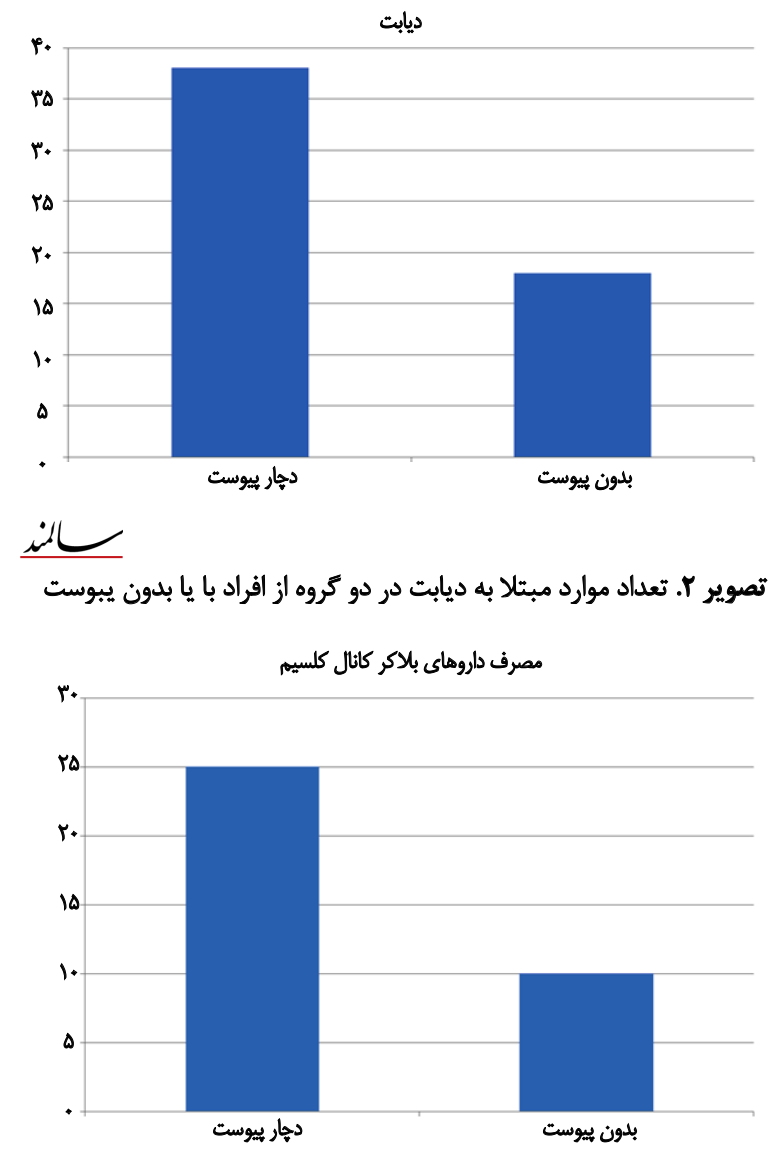

L

تصوير F. تعداد موارد مصرف كنينده داروهاى بلوك كنثند كانال كلسيم در دو

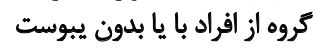

ضمن اينكه با توجه به نويابودن رشته تخصصى طب سالمندى،

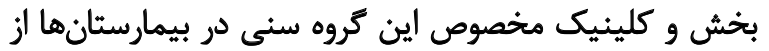

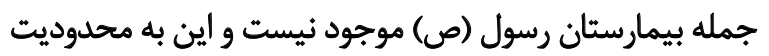

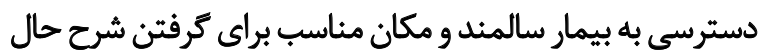

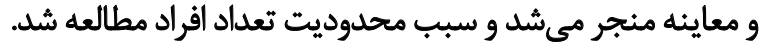

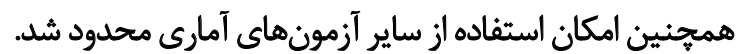

يافتها

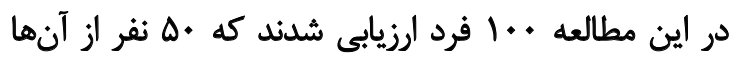

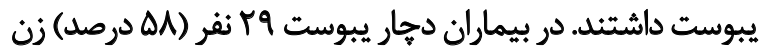

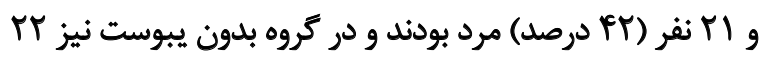

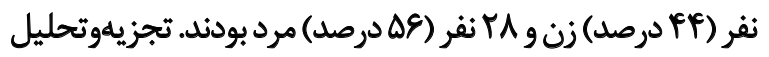

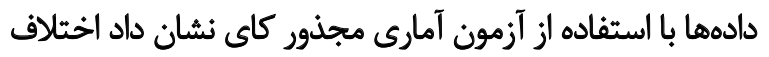

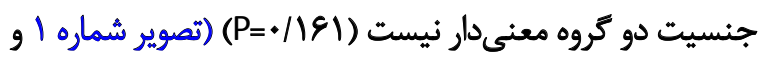

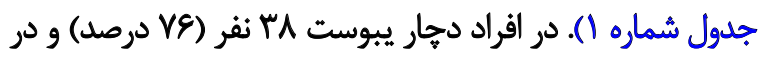

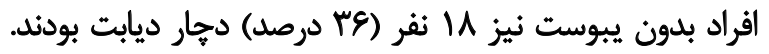

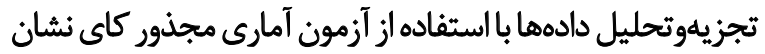

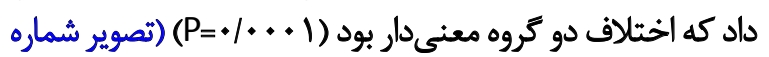

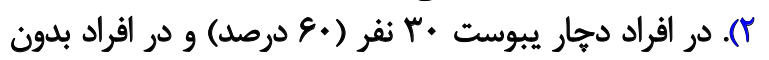
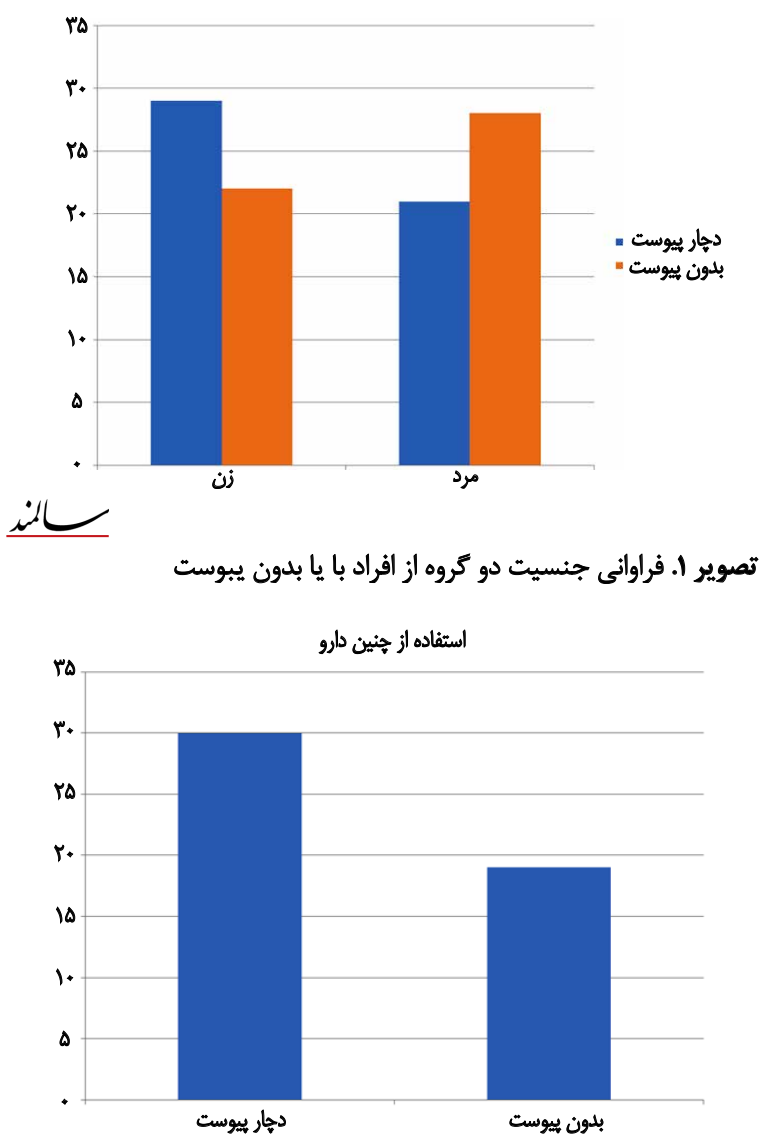<smiles>C1CC1C1CC1</smiles>

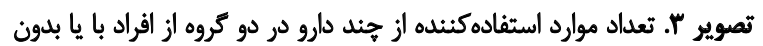
بيوسيث 


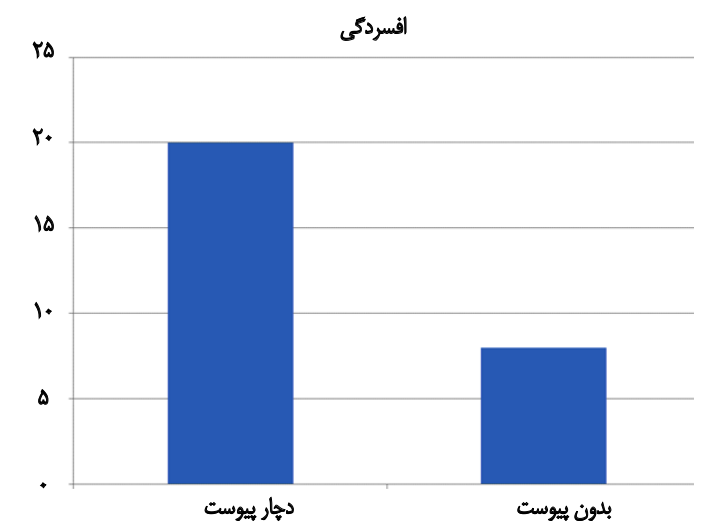

بالم

تصوير \&. تعداد موارد مبتلا به افسردكى در دو كروه از افراد با يا بدون يبوست
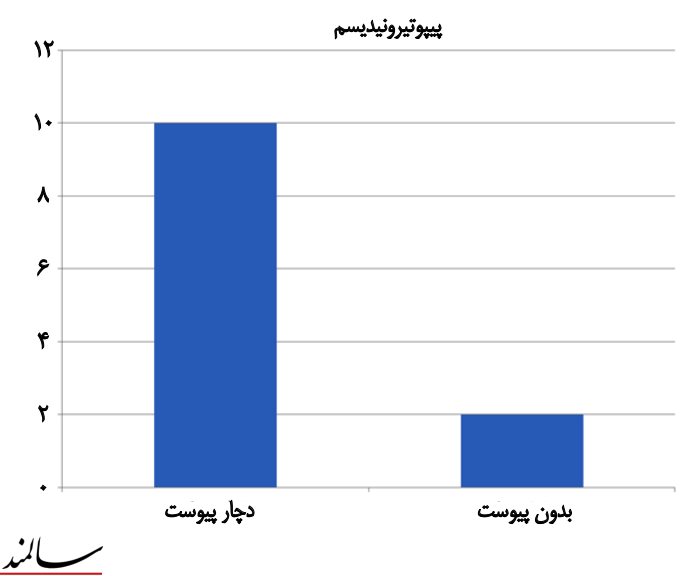

تصوير A. تعداد موارد مبتلا به هييوتيروئيديسم در دو كروه از افراد با يا بلدون يبوست بين دو كروه از اين نظر معنىدار است (P= (P. (تصوير)

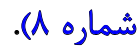

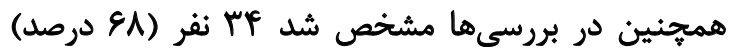

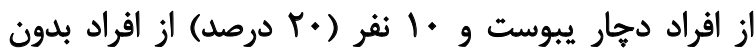

رزيم كم فيير

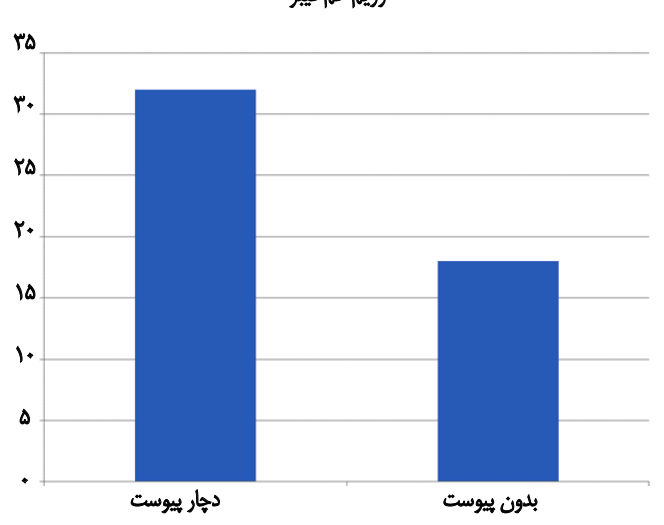

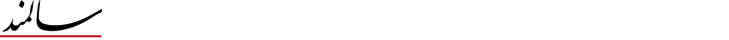

تصوير ثا. تعداد موارد استفادهكنثده از رذيم كمفيبر در دو كروه از افراد با

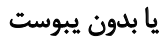
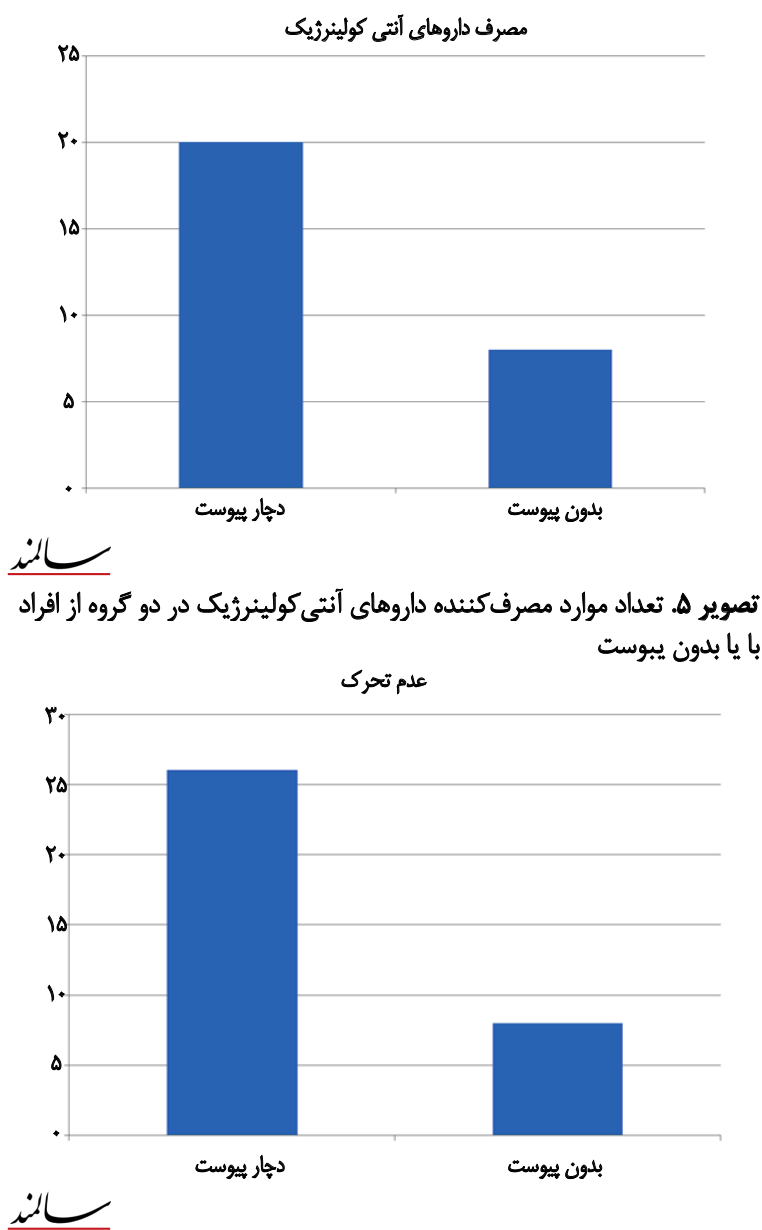

تصوير V. تعداد موارد دجار عدمتحرك در دو كروهاز افراد با يا بدون يبوست

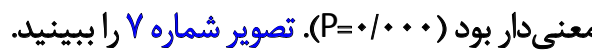

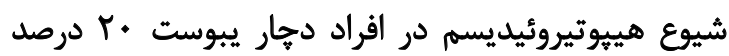

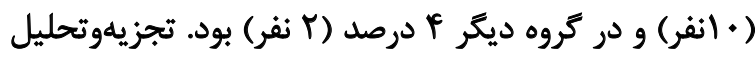

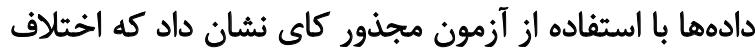

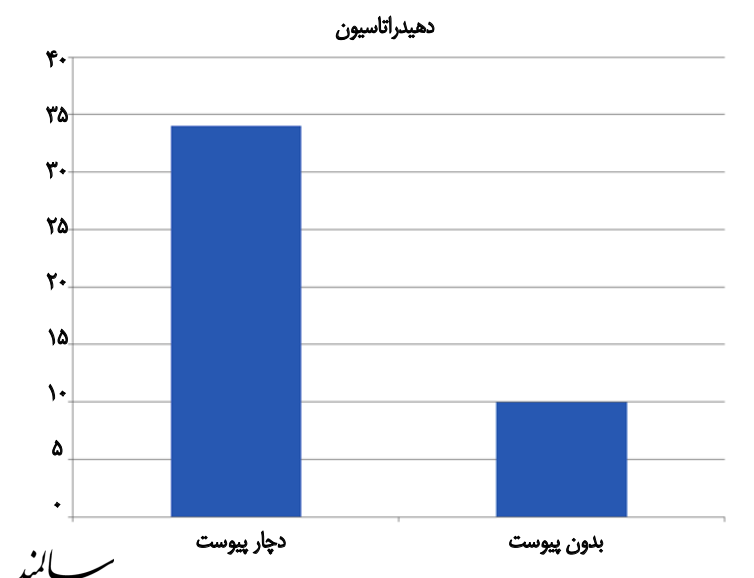

تصوير 9. تعداد موارد دجار دهيدراثأسيون در دو كروه از افراد با ها بدون يبوست 


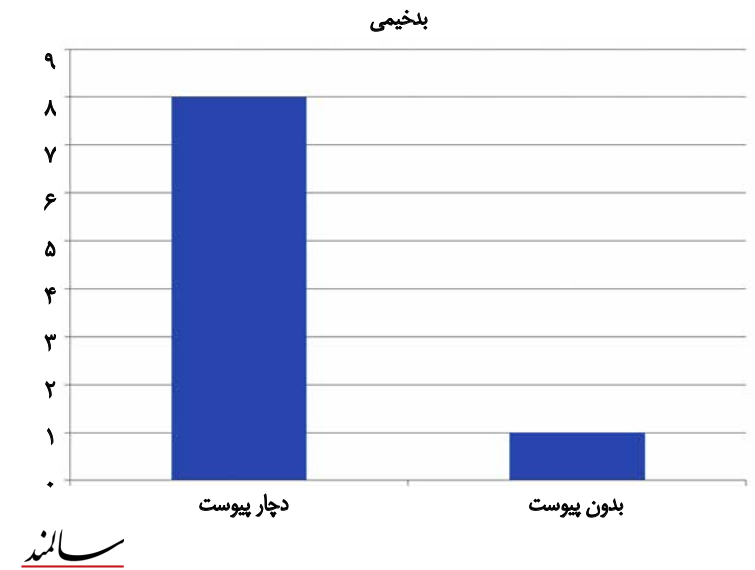

تصوير با. تعداد موارد مبتلا به بدخيمى در دو كروه از افراد با يا بدون يبوست
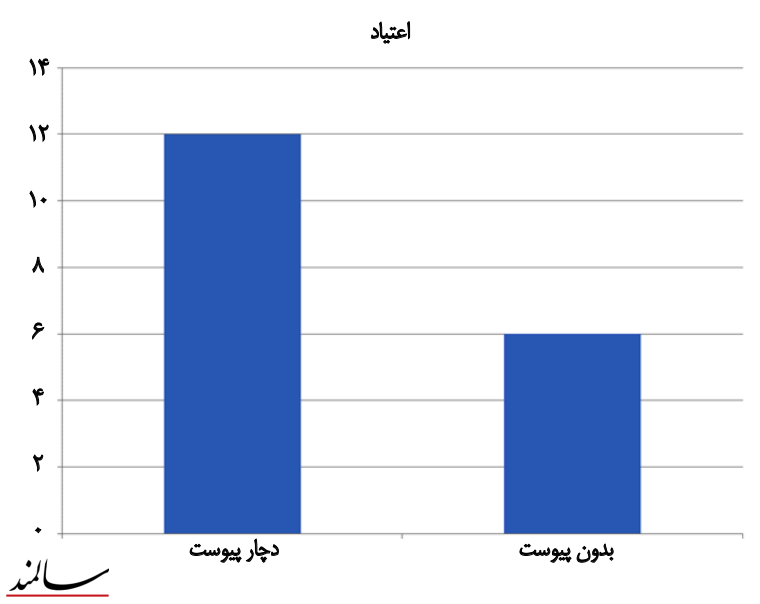

تصوير fا. تعداد موارد مبتلا به اعتياد در دو كروه از افراد با يا بدون يبوست

اعتياد داشتند، حال آنكه در زروه ديكر 9 نفر (rا دادرصد)

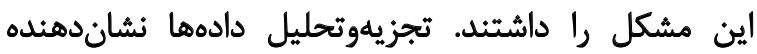

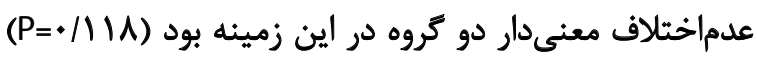

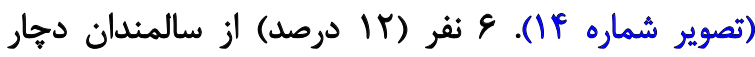

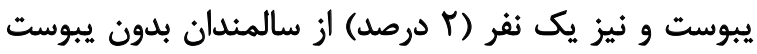

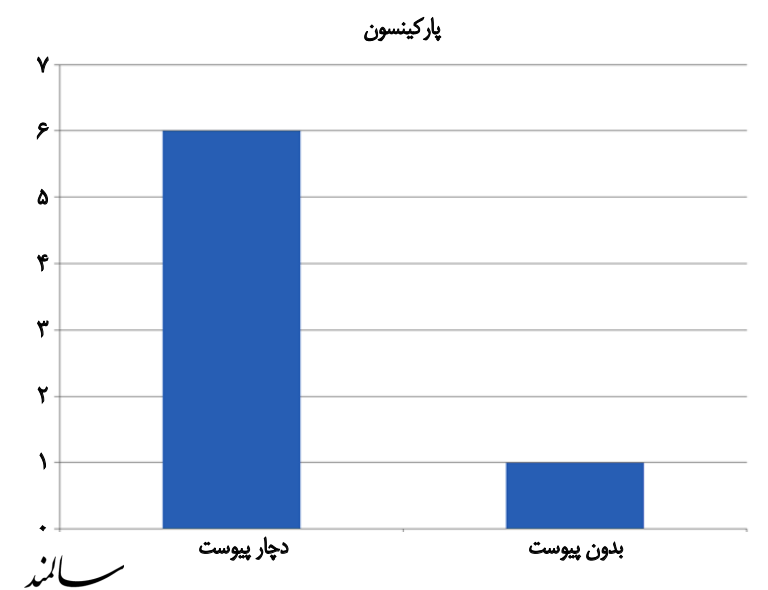

تصوير ها. تعداد موارد مبتلا به باركينسون در دو كروه از افراد با يا بلدون

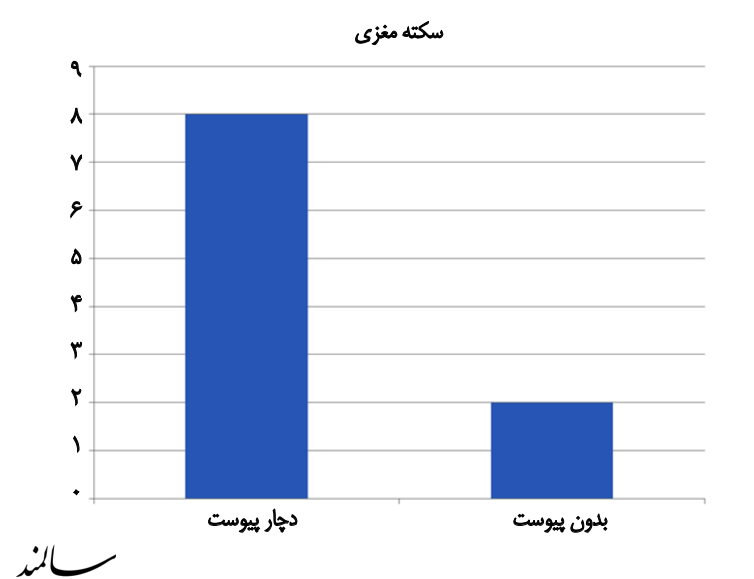

تصوير ال. تعداد موارد دجار سكته مغزى در دو كروه از افراد با يا بلدون يبوست

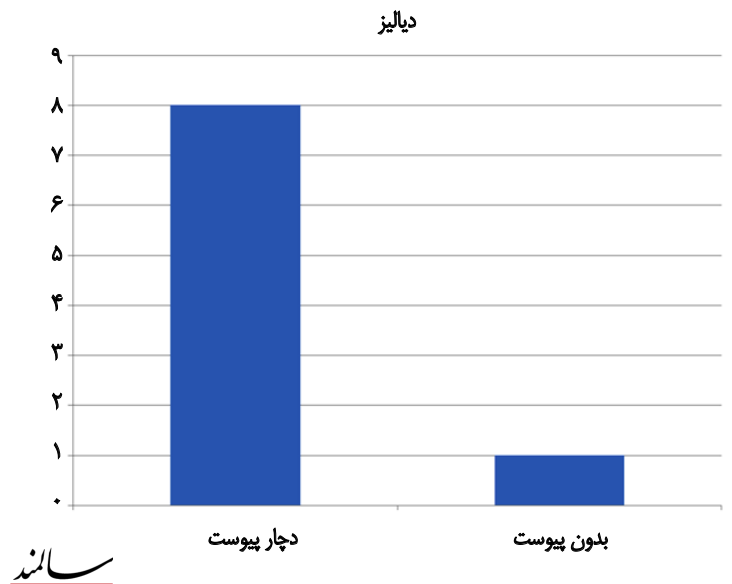

تصوير با. تعداد موارد دجار دياليز در دو كروه از افراد با يا بدون يبوست

يبوست در طول روز به ميزان كافى مايعات مصرف نمى كردند.

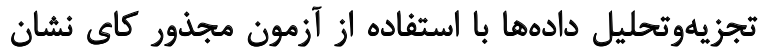

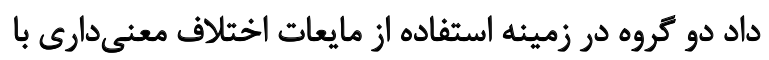

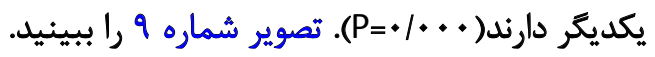

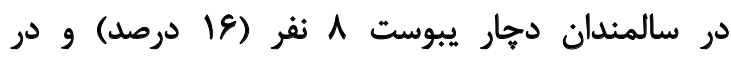

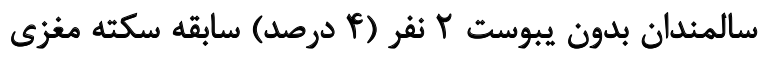

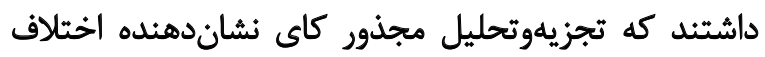

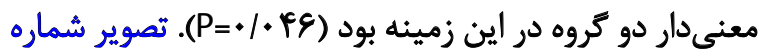

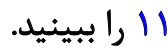

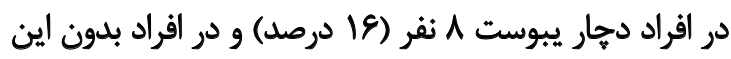

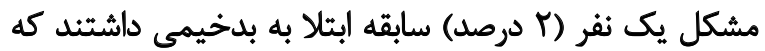

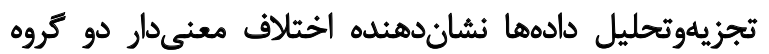

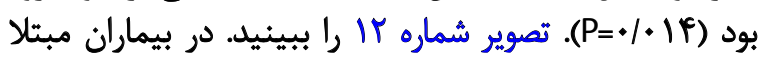

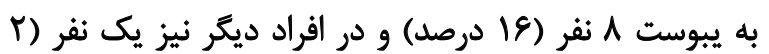

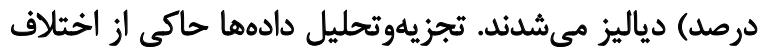

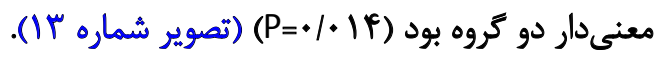

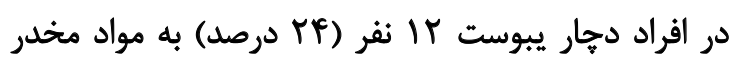


جدول ا. متغيرهاي بررسى شده در افراد با يا بدون يبوست

\begin{tabular}{|c|c|c|c|}
\hline$P$ & بدون ييوست & وهار يبوست & \\
\hline $.1 \ldots+1^{*}$ & $M$ & ra & ديابت \\
\hline.$/ \% \Lambda^{*}$ & 19 & r. & مصرف جند دارو \\
\hline..$++r^{*}$ & 1. & ra & مصرف كلسييه بلاكرها \\
\hline$+1+\Lambda^{*}$ & $\wedge$ & r. & مصرف أنتى كولينرزيكها \\
\hline $.1 \cdot A f$ & $f$ & 1. & أفسردگى \\
\hline$+\ldots+1^{*}$ & $\wedge$ & rq & عدم تحرى \\
\hline .1 .114 & r & 1. & هييوتيرونيديسم \\
\hline $.1 . . .1^{*}$ & 1. & me & دهيدراتاسيون \\
\hline$* / * \infty^{*}$ & M & m & رئيم كمفيبر \\
\hline 1.48 & r & $\wedge$ & سكته مغزى \\
\hline.$/+11 \%$ & 1 & $\wedge$ & بلخيمي \\
\hline $.1 .1 \%$ & 1 & $\wedge$ & 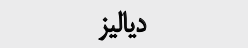 \\
\hline$+/ 11 \mathrm{~A}$ & 8 & ir & اعتياد \\
\hline $.1 \cdot 0 \cdot *$ & 1 & 8 & باركينسون \\
\hline
\end{tabular}

يبوست معمولاً بخشى طبيعى از عوارض افزايش سن به به شمار

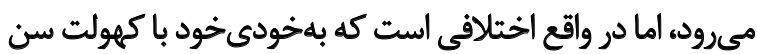

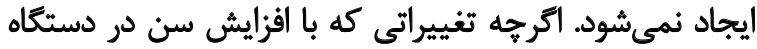

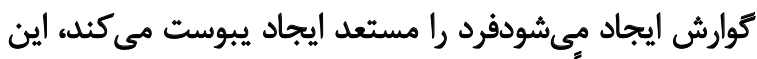

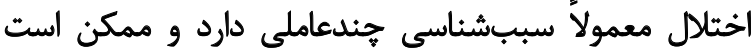

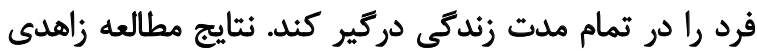

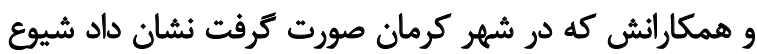

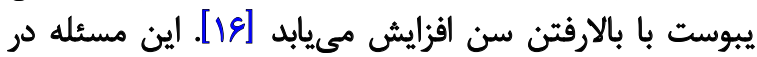

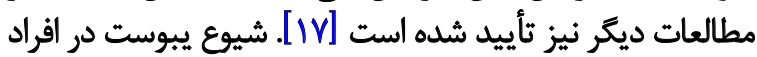

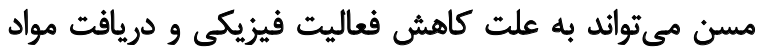

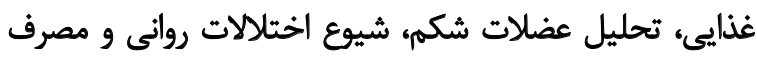

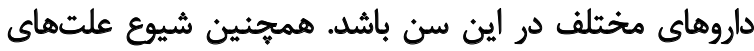
ثانويه نيز در افراد مسن افزايش مئيابد [1/]

r. عوامل متعددى ممكن است با إيجاد يبوست در ارتباط باشني

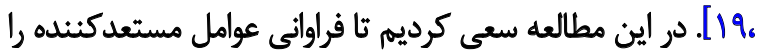

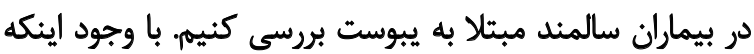

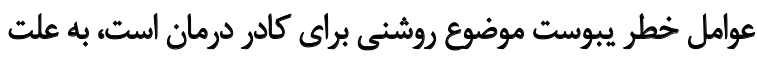

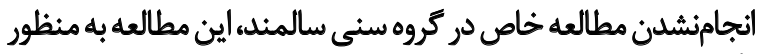

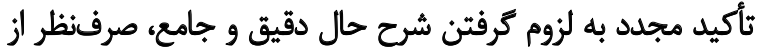

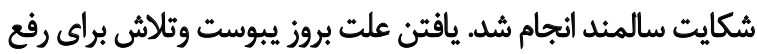

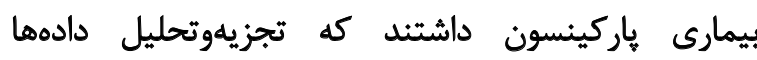

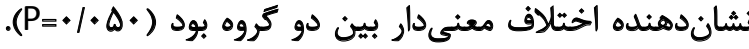
تصوير شماره ها إ إبينيد. يكى از مواردى كه در بيماران ارزيابي شد، ميزان دريافت

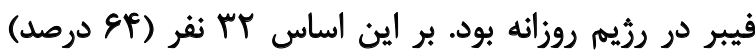

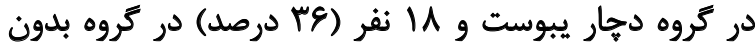

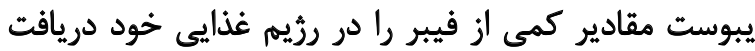

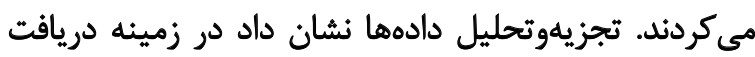

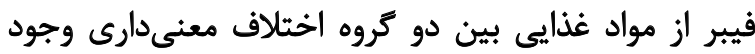

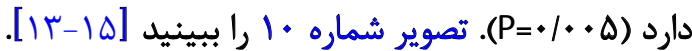

بحث

نتايج حاصل از مطالعه حاضر نشان داد فراوانى عواملى نظير

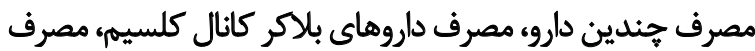

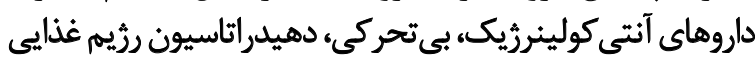

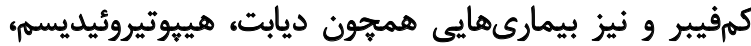

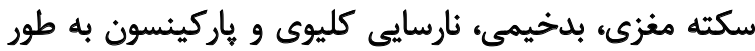

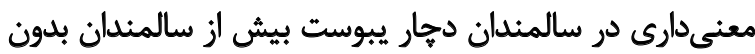

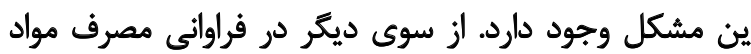

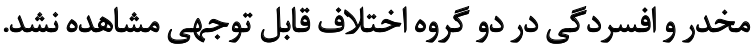


جدول r. داروهاي ايجادكثئله يبوست

\begin{tabular}{|c|c|}
\hline هيبيت مليتوس & بيمارىهاى اندوكرين و متابوليك \\
\hline 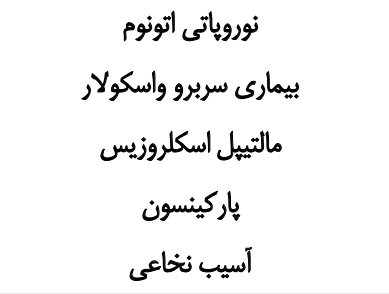 & بيمارى هاى ثورولوزيك \\
\hline اضسراب & اختالالات سايكولوزيك \\
\hline 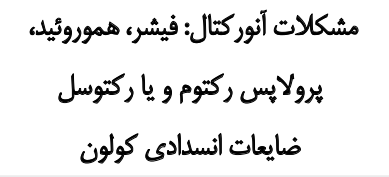 & ابنورماليتى هاى ساختمانى \\
\hline 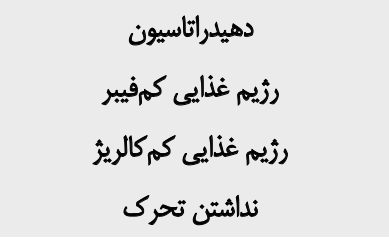 & سبك زندكى \\
\hline 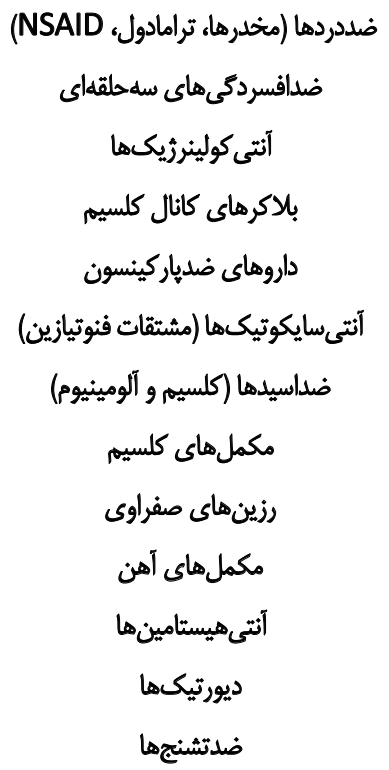 & داروها \\
\hline
\end{tabular}

估

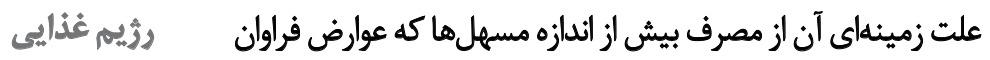

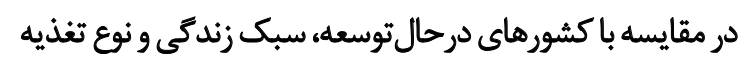

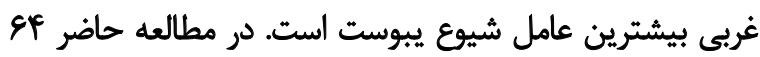

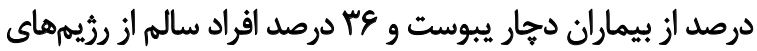

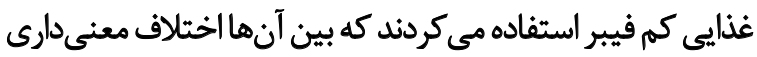

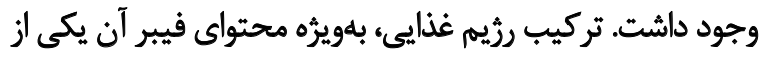

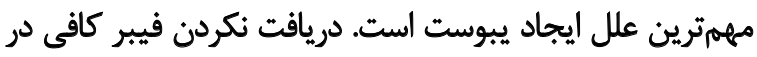

در سالمندان دارنده جلوكيرى خواهي كرد بيث.

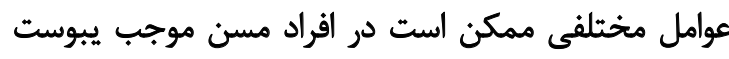

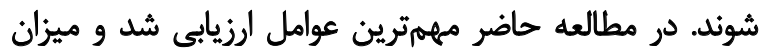

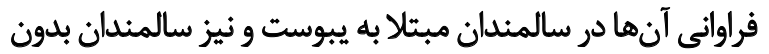

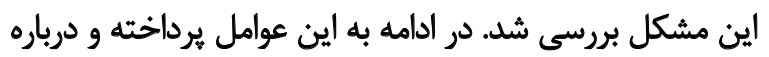
علت ايجاد يبوست بحث مىشود شرد 


\section{بيمارى هائ سيستميك}

بيمارىهاى سيستميك نظير ديابت مليتوس، مالتيّل

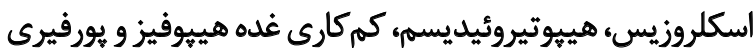

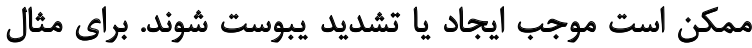

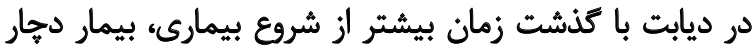

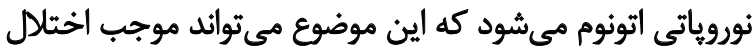

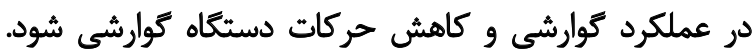

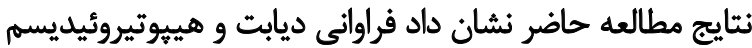

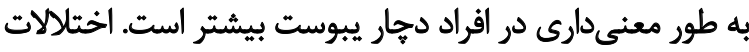

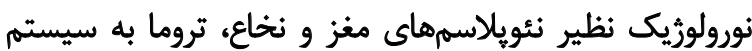

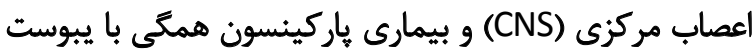

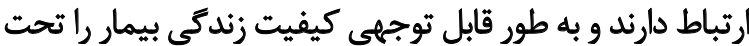

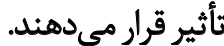

\section{نتيجه تيرى نهايى}

شناخت عوامل مؤثر در بروز يبوست در جمعيت سالمندان و ورائ

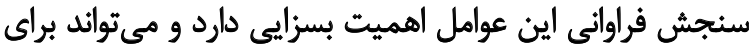

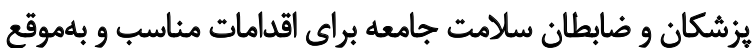

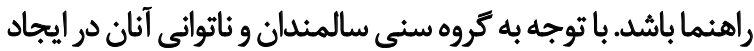

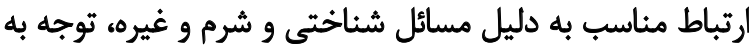

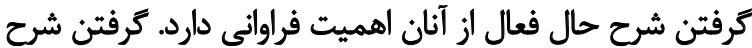

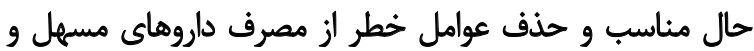
بروز عوارض يبوست در كروه آسيبيذير سالمندان تا حئ حد زيادي جلوكيرى مي كند.

در اين مطالعه فراوانى عواملى نظير مصرف جند ميند دارو، مصرف

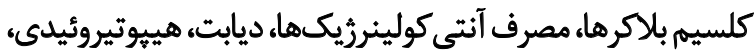

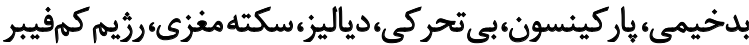

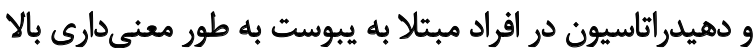

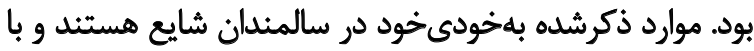

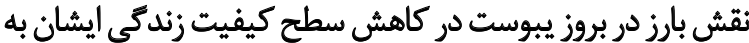

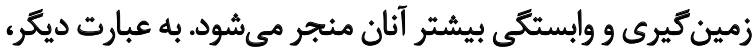

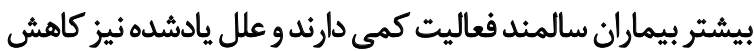

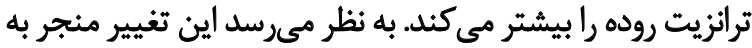

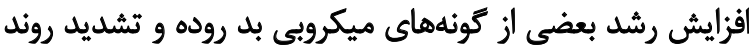

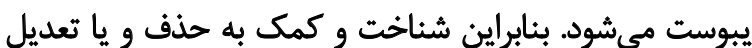
اين عوامل خطر به كاهش بار اقتصادى درمان كمك قابل توجيه بنهي

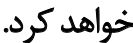

$$
\text { تشكر و قدروانئي }
$$

اين مقاله از يايان نامه تخصصى يزشكى خانم راحله على علىمرادزاده

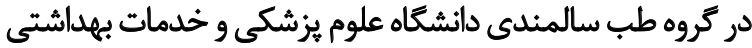
درمانى ايران كرفته شده است.
رزيم غذايى موجب ايجاد مدفوعى ميىشود كه حجم كمى دارد،

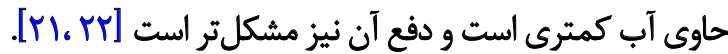
در جوامعى نظير آفريقاى غربى كه در آن به طور ميانكين در

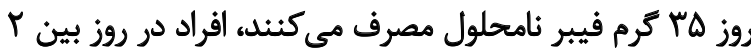

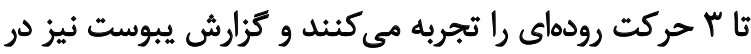

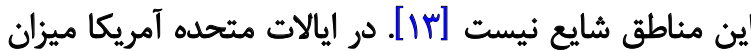

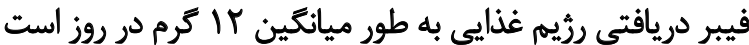

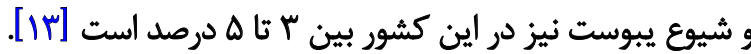

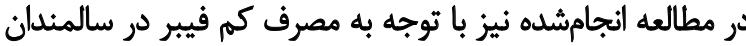

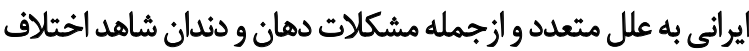

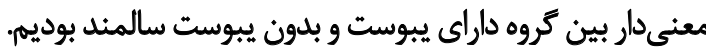

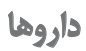
داروهاي متعددى نظير آنتى كولينرزيكها، ضد افسردگى هاو و مخدرها ممكن است به وسيله ممانعت از سيكنال هاي نوريك نوروني

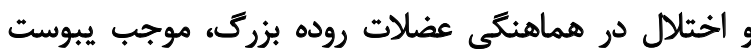

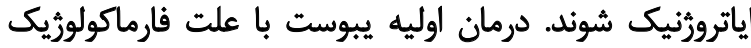
مشخص، شامل قطع دارو و در صورت امكان، جايكزينى آن بانيا

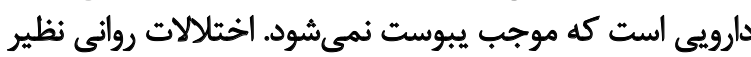

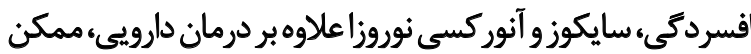

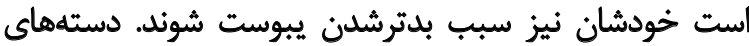

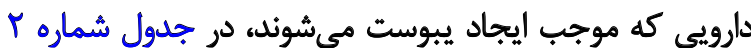

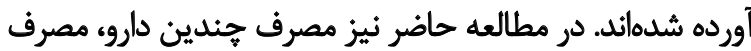

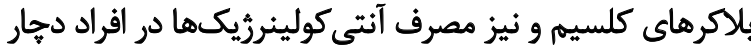

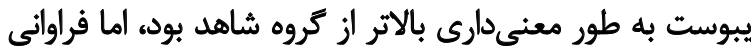

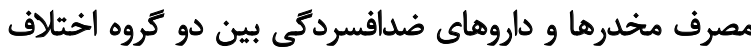

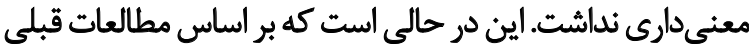

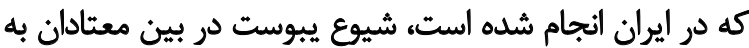

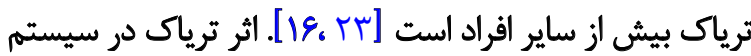

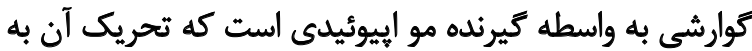

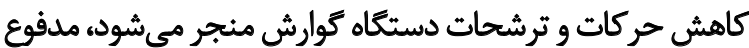

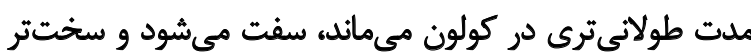

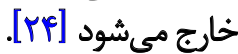

بلدخيمىها

نئويلاسمهاى كولون، ركتوم و مقعد در حين بزركترشدن

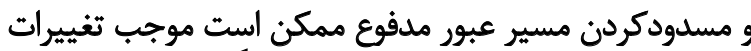

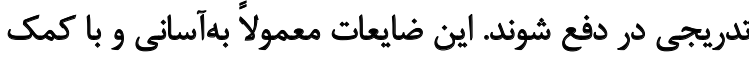

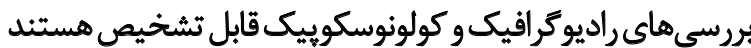

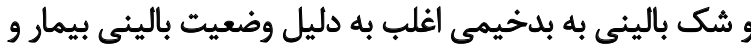

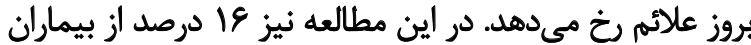

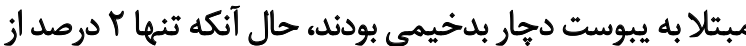

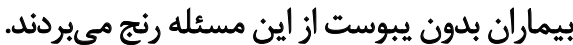




\section{References}

[1] Singh G, Lingala V, Wang H, Vadhavkar S, Kahler KH, Mithal A, et al. Use of health care resources and cost of care for adults with constipation. Clinical Gastroenterology and Hepatology. 2007; 5(9):1053-8. doi: 10.1016/j.cgh.2007.04.019

[2] Chong PS, Bartolo DCC. Hemorrhoids and fissure in ano. Gastroenterology Clinics of North America. 2008; 37(3):627-44. doi: 10.1016/j.gtc.2008.07.001

[3] Huang WS, Wang CS, Hsieh CC, Lin PY, Chin CC, Wang JY Management of patients with stercoral perforation of the sigmoid colon: Report of five cases. World Journal of Gastroenterology. 2006; 12(3):500-503 doi: 10.3748/wjg.v12.i3.500

[4] Singh G, Kahler KH, Bharathi V, et al. Constipation in adults: Complications and comorbidities. Gastroenterology 2005; 128: A154-A154.

[5] De Schryver AM, Keulemans YC, Peters HP, Akkermans LM, Smout AJ, De Vries WR, et al. Effects of regular physical activity on defecation pattern in middle-aged patients complaining of chronic constipation. Scandinavian Journal of Gastroenterology. 2005 ;40(4):422-9. doi: 10.1080/00365520510011641

[6] Tiainen K, Hurme M, Hervonen A, Luukkaala T, Jylha M. Inflammatory markers and physical performance among nonagenarians. The Journals of Gerontology Series A: Biological Sciences and Medical Sciences. 2010; 65A(6):658-63. doi: 10.1093/gerona/ glq056 (28)

[7] Halter J, Ouslander J, Tinetti M, Studenski S, High K, Asthana S. Hazzard's geriatric medicine and gerontology. New York: McGraw-Hill Education; 2008

[8] Leung FW. Etiologic factors of chronic constipation-Review of the scientific evidence. Digestive Diseases and Sciences. 2007 ;52(2):313-6. doi: 10.1007/s10620-006-9298-7

[9] Zahedi MJ, Moghadam SD, Abbasi MH, Mirzaei SM. [The assessment prevalence of functional constipation and associated factors in adults: A community-based study from Kerman, southeast, Iran (2011-2012) (Persian)]. Govaresh. 2014; 19(2):95-101.

[10] Guigoz Y, Doré J, Schiffrin EJ. The inflammatory status of old age can be nurtured from the intestinal environment. Current Opinion in Clinical Nutrition and Metabolic Care. 2008; 11(1):1320. doi: $10.1097 /$ mco.0b013e3282f2bfdf

[11] Suares NC, Ford AC. Prevalence of, and risk factors for, chronic idiopathic constipation in the community: systematic review and meta-analysis. American Journal of Gastroenterology. 2011; 106(9):1582-91. doi: 10.1038/ajg.2011.164

[12] Kahle-Wrobleski K, Corrada MM, Li B, Kawas CH. Sensitivity and specificity of the mini-mental state examination for identifying dementia in the oldest-old: the 90+ study. Journal of the American Geriatrics Society. 2007; 55(2):284-9. doi: 10.1111/j.15325415.2007.01049.x (32)

[13] Singh T, Newman AB. Inflammatory markers in population studies of aging. Ageing Research Reviews. 2011; 10(3):319-29. doi: 10.1016/j.arr.2010.11.002

[14] Jeon MJ, Chung SM, Jung HJ, Kim SK, Bai SW. Risk factors for the recurrence of pelvic organ prolapse. Gynecologic and Obstetric Investigation. 2008;66(4):268-73. doi: 10.1159/000149851
[15] Ouwehand AC, Tiihonen K, Saarinen M, Putaala H, Rautonen N. Influence of a combination of Lactobacillus acidophilus NCFM and lactitol on healthy elderly: Intestinal and immune parameters. British Journal of Nutrition. 2008;101(03):367. doi: 10.1017/ s0007114508003097

[16] Koch T, Hudson S. Older people and laxative use: literature review and pilot study report. Journal of Clinical Nursing. 2000 ;9(4):516-25. doi: 10.1046/j.1365-2702.2000.00357.x

[17] Deveaux PJ, Galandiuk S. Etiology of acquired colorectal disease: Constipation. Constipation. 15-23. doi: 10.1007/978-184628-275-1_3

[18] Pappagallo M. Incidence, prevalence, and management of opioid bowel dysfunction. American Journal of Surgery. 2001; 182(5):11-8. doi: 10.1016/s0002-9610(01)00782-6

[19] Panchal SJ, Müller-Schwefe P, Wurzelmann JI. Opioid-induced bowel dysfunction: prevalence, pathophysiology and burden. International Journal of Clinical Practice. 2007; 61(7):1181-7. doi: 10.1111/j.1742-1241.2007.01415.x

[20] Opal SM, DePalo VA. Anti-inflammatory cytokines. Chest. 2000; 117(4):1162-72. doi: 10.1378/chest.117.4.1162

[21] Hayashi H, Sakamoto M, Kitahara M, Benno Y. Molecular analysis of fecal microbiota in elderly individuals using $16 \mathrm{~s}$ rdna library and T-rflp. Microbiology and Immunology. 2003; 47(8):55770. doi: 10.1111/j.1348-0421.2003.tb03418

[22] Wood IS, Trayhurn P. Signalling role of adipose tissue: Adipokines and inflammation in obesity. Biochemical Society Transactions. 2005; 33(5):1078. doi: 10.1042/bst20051078 (27)

[23] Cesari M. Inflammatory markers and onset of cardiovascular events: results from the health $\mathrm{ABC}$ study. Circulation. 2003; 108(19):2317-22. doi: 10.1161/01.cir.0000097109.90783.fc

[24] Cesari M, Penninx BWJ, Newman AB, Kritchevsky SB, Nicklas BJ, Sutton-Tyrrell K, et al. Inflammatory markers and cardiovascular disease (The Health, Aging and Body Composition [Health ABC] Study). The American Journal of Cardiology. 2003; 92(5):522-8. doi: 10.1016/s0002-9149(03)00718-5 